\title{
Uso de Dispositivos Móveis como Recurso Didático na Formação Docente
}

\author{
Gabriela Cunha de Oliveira ${ }^{1}$, Nadma Farias Kunrath ${ }^{1}$, Sérgio Antônio Francalino ${ }^{1}$ \\ Rocha, Luiz Augusto Matos da Silva ${ }^{1}$
}

\author{
${ }^{1}$ Pós-Graduação Lato Sensu em Tecnologias da Informação e Comunicação - \\ Universidade Federal do Acre (UFAC) - CEP 69.915-900 - Rio Branco- AC, Brasil \\ gabriela.oliveira@uninorteac.edu.br, nadfak@gmail.com, \\ sergio.francalino@gmail.com, luiz.matos@ufac.br
}

\begin{abstract}
Resumo. Este trabalho trata- se de uma pesquisa-ação realizada com alunos de Licenciatura em Matemática, com o objetivo de avaliar a percepção e aptidão dos participantes quanto à utilização das Tecnologias da Informação e Comunicação (TIC) em dispositivos móveis como recurso didático. $O$ método foi dividido em três partes: diagnóstico inicial, oficina e avaliação final. Além de o trabalho propor um método de ação para a formação docente, concluiu- se que estudantes de licenciatura em matemática se apresentam aptos e engajados a utilizarem dispositivos móveis nas suas futuras práticas pedagógicas.
\end{abstract}

Abstract. This work is an action research carried out with students of Mathematics Degree, with the objective of evaluating the participants' perception and aptitude regarding the use of Information and Communication Technologies (ICT) in mobile devices as a didactic resource. The method was divided into three parts: initial diagnosis, workshop and final evaluation. In addition to proposing a method of action for teacher training, it was concluded that undergraduate students in mathematics are apt and engaged to use mobile devices in their future pedagogical practices.

\section{Introdução}

A sociedade atual considerada "Sociedade da Informação", que dispõe de tecnologias para o compartilhamento da cultura e do conhecimento, está em constante busca pela democratização do acesso aos recursos da Tecnologia da Informação e Comunicação (TIC). Além disso é cada vez maior o número de crianças e adolescentes que convivem com as tecnologias digitais, especialmente os dispositivos móveis [Cetic, 2015]. Assim, a ascensão de tais tecnologias tem despertado estudos voltados ao uso das TIC no contexto educacional.

O uso de recursos tecnológicos como ferramentas didáticas na escola já é uma realidade no Brasil e no mundo. Projetos de inclusão digital na escola com aquisição de projetores, lousas digitais, implantação de laboratórios de informática com acesso à internet, dentre outros, são exemplos de iniciativas existentes que visam aproximação do processo de ensinar e aprender com a tecnologia. Entretanto, a utilização de recursos tecnológicos móveis no processo educativo (mobile learning) ainda carece de aceitação por parte de alguns professores e sistemas de ensino. Além da resistência desses 
profissionais em inovar no modo de ensinar [Ribeiro, 2013], há ainda alguns estados no Brasil que se proíbe o uso de tais dispositivos nas dependências escolares. E ainda, a formação de professores caracteriza-se deficiente, aponta Ferreira (2015), onde explica que professores vão para as salas de aula com instrumentos intelectuais ultrapassados, acarretando a falta de estímulo dos discentes, especialmente no ensino da matemática, que tem apontado fracassos por diversos fatores.

Diante disso e da necessidade de analisar o mobile learning a partir da percepção docente [Nascimento e Filho, 2016], propôs-se um trabalho com o objetivo de avaliar a percepção e aptidão de estudantes de licenciatura quanto à utilização de aplicativos em dispositivos móveis com vistas a responder a seguinte pergunta: alunos de licenciatura estão aptos e engajados para utilizar as TICs em dispositivos móveis em suas futuras práticas pedagógicas no ensino da matemática?

O restante deste texto está organizado conforme descrito a seguir. A Seção 2 apresenta os principais conceitos de Informática na Educação e Mobile Learning. A Seção 3 descreve a metodologia utilizada nesta pesquisa. A Seção 4 apresenta as etapas da ação da pesquisa. Na seção 5 os resultados e discussões e por fim, a Seção 6 traz as considerações finais.

\section{Informática na Educação}

No contexto educacional atual, onde a tecnologias está cada vez mais presente na vida dos estudantes, os atos de ensinar e aprender na escola passa a ter um novo formato. Diferente da escola tradicional, que, só quem detinha o conhecimento era o professor e os alunos eram somente receptores do que o professor transmitia, hoje em dia o cenário não é mais o mesmo.

Para Devalli e Corrêa (2014), a aplicação da informática nos métodos de ensino atuais vem mudando significativamente esse cenário do tradicionalismo e o uso das tecnologias na aprendizagem torna o ambiente de ensino mais dinâmico, estimulando o interesse de todas as partes e a troca de informações. Ao comparar ambientes em que a tecnologia não é instrumento pedagógico, com ambientes em que a tecnologia está inserida, é possível ver claramente a diferença entre o nível e o aproveitamento do aprendizado. Assim, a percepção da oportunidade das tecnologias digitais no meio pedagógico pode ser um caminho para a melhoria do desempenho dos alunos [Souza, A. Murta, C. e Leite, L. 2016].

Além disso, já existe uma quantidade considerável de objetos educacionais, ferramentas e aplicativos, livres e gratuitos voltados aos diferentes níveis e temas de ensino. Pesquisa de Slomp e Machado (2013), por exemplo, aponta o mapeamento e avaliação de 305 aplicativos educacionais livres para dispositivos móveis com finalidades educacionais.

Havendo a disponibilidade no mercado dessas tecnologias voltadas à educação, cabe ao professor a escolha da ferramenta e da proposta metodológica de ensino. Além disso, a utilização de softwares em sala de aula deve ser norteada por interesses pedagógicos e com isso a mediação do professor desempenha um papel importantíssimo e determinante [Alves, E.; Assis, C. e Martins, F. 2015]. 
VI Congresso Brasileiro de Informática na Educação (CBIE 2017)

Anais do XXIII Workshop de Informática na Escola (WIE 2017)

\subsection{Mobile Learning}

Os celulares Smartphones conectados à internet estão cada vez mais presentes na vida de crianças e adolescentes. Pesquisas do Cetic (2015) apontam que 73\% dos alunos utilizam o celular como o principal equipamento para acessar a internet (figura 1).

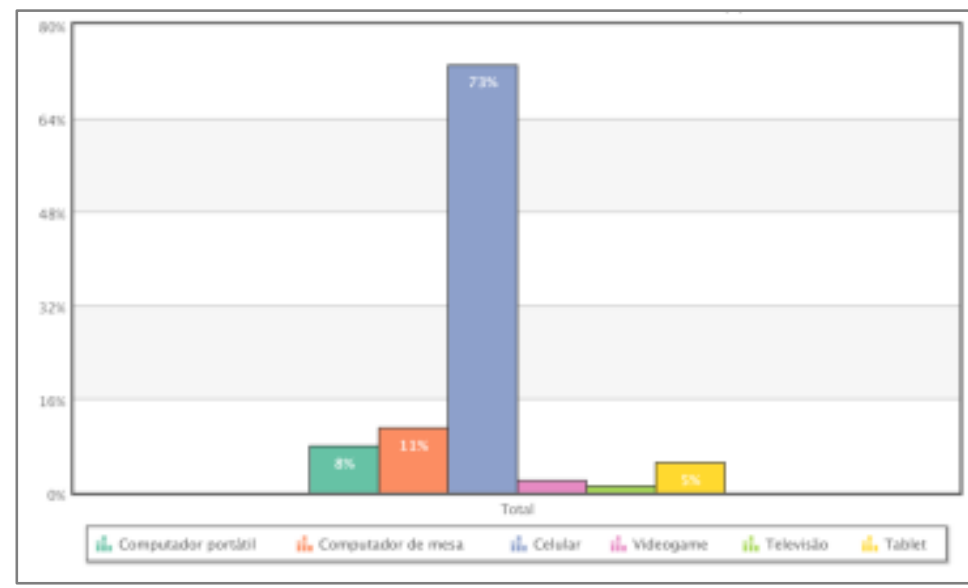

Figura1. Proporção de alunos por principal equipamento para acess ar a internet

Fonte: CETIC, 2015

Com o aumento do poder computacional presente em tais dispositivos, pesquisas têm sido feitas com vistas a difundir o seu potencial e uso no contexto educacional. Assim, a utilização de dispositivos móveis na educação criou um novo conceito denominado mobile learning [Arantes e Seabra, 2016], termo utilizado para referir-se a utilização de tecnologias de rede sem fio para difundir o ensino e aprendizagem [Oliveira e Medina, 2007].

$\mathrm{O}$ m-learning surge como uma alternativa de ensino e treinamento com os objetivos de: executar tarefas, consultar informações via internet, registrar fatos pela câmera ou áudios, acessar conteúdos a qualquer momento, expandir as estratégias de aprendizagem formal e informal [Marçal, E.; Andrade, R. e Rios, R. 2015], favorecendo a educação quando há mediação docente e objetivo pedagógico claro [Nascimento e Filho, 2016].

Entretanto, ainda que esse potencial seja visível, existem sistemas de ensino, governos e professores que, de modo geral não aceitam tais dispositivos na escola. Em alguns estados e cidades do país, existem leis proibitivas do uso do celular na escola. É o caso da Lei Estadual 3109 [Acre, 2015] que proíbe o uso de dispositivos móveis (smartphones e tablets) nas dependências escolares.

\section{Método da Pesquisa}

Quanto aos procedimentos do trabalho, o tipo da pesquisa adotada foi a pesquisa ação, na qual identificou- se o problema da pesquisa e o perfil do público alvo; promoveu- se uma ação aos participantes e colheu- se os resultados da ação. Nesse tipo de pesquisa, os participantes agem ativamente na investigação do trabalho. É o que explica Baldissera (2011) quando expõe que uma pesquisa pode ser qualificada de pesquisa ação quando houver realmente uma ação por parte das pessoas implicadas no processo 
investigativo. Utilizou- se também a Survey, um tipo de procedimento que busca dados e informações diretamente com o público alvo através de questionário.

Os Participantes da pesquisa foram 35 estudantes do curso de licenciatura em Matemática da Universidade Federal do Acre. Além disso, a presente pesquisa foi desenvolvida em 3 etapas: diagnóstico inicial, oficina e avaliação final.

O diagnóstico inicial deu- se a partir de um questionário composto por 14 questões aplicadas aos 35 participantes a fim de subsidiar o planejamento e organização da ação e identificar o perfil dos participantes, quanto as suas informações demográficas, quanto ao conhecimento e experiências sobre os aplicativos móveis como recurso didático de modo geral, e quanto ao conhecimento e utilização de uma listagem de 17 aplicativos para dispositivos móveis, sendo 10 voltados ao ensino da matemática e 7 aplicativos de apoio (para correção de questões, apresentação de aulas etc.).

A Oficina, ação da pesquisa constituiu-se de uma apresentação sobre os conceitos principais do Mobile Learning; da exposição das funcionalidades e principais características de 6 aplicativos escolhidos dentre os listados diagnóstico inicial e de atividades práticas com os participantes utilizando tais aplicativos. $\mathrm{O}$ critério para a escolha dos aplicativos foi a maior porcentagem de participantes que conheciam os aplicativos, a disponibilidade dos mesmos para o sistema android e ainda, uma avaliação geral dos aplicativos pelos autores da pesquisa. Para a escolha dos aplicativos de apoio, além dos anteriores, utilizou- se o critério de que os aplicativos não tivessem as mesmas funcionalidades.

E a avaliação final, feita através de um segundo questionário, no qual foram avaliadas a utilidade e facilidade dos aplicativos e os indícios de impactos positivos ou negativos na percepção e motivação dos participantes para utilizar dispositivos móveis como recurso didático em suas futuras práticas pedagógicas.

\section{Utilização de Dispositivos Móveis em Sala de Aula}

A oficina intitulada "Utilização de Dispositivos Móveis em Sala de Aula" aconteceu em 2 dias (7 e 9 de dezembro de 2016), na carga horária total de 8 horas com a participação de 24 participantes dos 35 que responderam o diagnóstico inicial.

\subsection{Perfil dos Participantes}

A maioria dos participantes (69\%) são do sexo masculino e $66 \%$ na faixa etária de 16 a 20 anos. $40 \%$ dos participantes cursam matemática por afinidade à docência e $34 \%$ por afinidade ao curso de matemática. Além disso, 97\% afirmam que utilizariam aplicativos, jogos e outras ferramentas tecnológicas em sala de aula com os seus futuros alunos. E ainda, 60\% dos participantes afirmam já terem participado de atividades de extensão voltadas às TICS no ensino da matemática. A maioria dos participantes $(66 \%)$ durante o seu ensino básico não tiveram professores que utilizaram ferramentas tecnológicas no ensino, enquanto $34 \%$ tiveram essa experiência. Já atualmente no ensino superior, $66 \%$ dos seus professores utilizam ou já utilizaram ferramentas tecnológicas na sala de aula. Quanto ao conhecimento da Lei ${ }^{\circ} 3.109$ de 29/12/2015, que trata da proibição do uso de celulares nas escolas do Estado do Acre 54\% não conheciam a lei. 
Quanto ao conhecimento sobre os 17 aplicativos listados no questionário inicial, os aplicativos mais conhecidos voltados ao ensino da matemática foram: o Geogebra ${ }^{1}$ com 63\%, o Malmath ${ }^{2}$ com 14\% e o Math Academy ${ }^{3}$ com 17\% sendo estes os aplicativos de matemática escolhidos para serem utilizados na Oficina. Quanto aos aplicativos de apoio, somente o Nearpod já tinha sido utilizado pelos participantes $(6 \%)$. Além do Nearpod ${ }^{4}$ escolheu- se o Plickers $^{5}$ e o Share it ${ }^{6}$ para compor os aplicativos de apoio.

A oficina foi ministrada em 4 etapas: explanação inicial sobre tecnologias na educação, tecnologias no ensino da matemática e m-learning; apresentação e experimentação dos aplicativos voltados a matemática; atividade prática e, por fim, demonstração dos aplicativos de apoio.

\subsection{Explanação inicial}

$\mathrm{Na}$ explanação inicial foram apresentados os principais conceitos, dados estatísticos e vídeos sobre a evolução das tecnologias educacionais, inovações e grupos de pesquisa que envolve tecnologias no ensino da matemática e os fundamentos e aplicações do $m$ learning, conforme os temas abordados no referencial teórico deste trabalho e na sequência, as leis proibitivas do uso celular na escola vigente em alguns estados/cidades do país. Parte dessa apresentação foi realizada por meio de um retroprojetor e outra parte com auxílio de um dos aplicativos de apoio - Nearpod - no qual permite, através de um código criado pelo aplicativo, que os alunos visualizassem em tempo real a apresentação do professor - slides - em seus dispositivos móveis.

\subsection{Apresentação e experimentação dos aplicativos}

Após a explanação inicial, foram apresentados aos alunos os aplicativos de matemática a serem trabalhados na oficina (Geogebra, Math Academy e MalMath), com suas respectivas definições, principais funcionalidades, opções de uso, bem como o potencial de seus benefícios pedagógicos.

$\mathrm{Na}$ sequência da programação, foi proposto aos alunos que acessassem e utilizassem, por cerca de uma hora, os aplicativos explorando os seus recursos. Nessa etapa os alunos ficaram livres para interagir entre eles e com os instrutores da oficina (figura 2).

\footnotetext{
${ }^{1} \mathrm{https}: / /$ www.geogebra.org/

${ }^{2} \mathrm{http}: / /$ www.malmath.com/

${ }^{3} \mathrm{http}: / /$ www.mathacademygame.com/pt

${ }^{4}$ https://nearpod.com/

${ }^{5} \mathrm{https}: / /$ plickers.com/

${ }^{6} \mathrm{http}: / /$ www.ushareit.com/
} 
VI Congresso Brasileiro de Informática na Educação (CBIE 2017)

Anais do XXIII Workshop de Informática na Escola (WIE 2017)

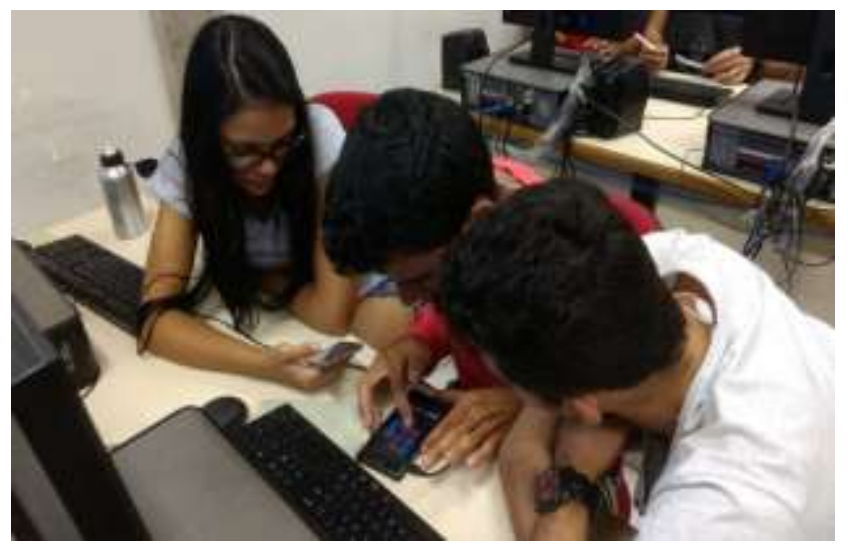

Figura 2. Participantes da oficina explorando os aplicativos

\subsection{Atividade prática}

Após a exploração dos aplicativos, foi proposto aos alunos que formassem equipes de 3 pessoas, escolhessem um dos aplicativos, produzissem e apresentassem para a turma uma atividade a ser solucionada utilizando o aplicativo escolhido. Além disso, deveriam elaborar um plano de aula, sendo a atividade, o conteúdo da aula. Nessa etapa, os alunos ficaram livres para escolher um tema e criar uma atividade, escolhendo o nível de ensino e a forma de apresentação para a turma (figura 3). Além do proposto, o plano de aula deveria ser elaborado e compartilhado no Website redenovaescolaclube.org.brcomunidade online de educação, que tem como uma de suas funcionalidades, o compartilhamento de planos de aula.

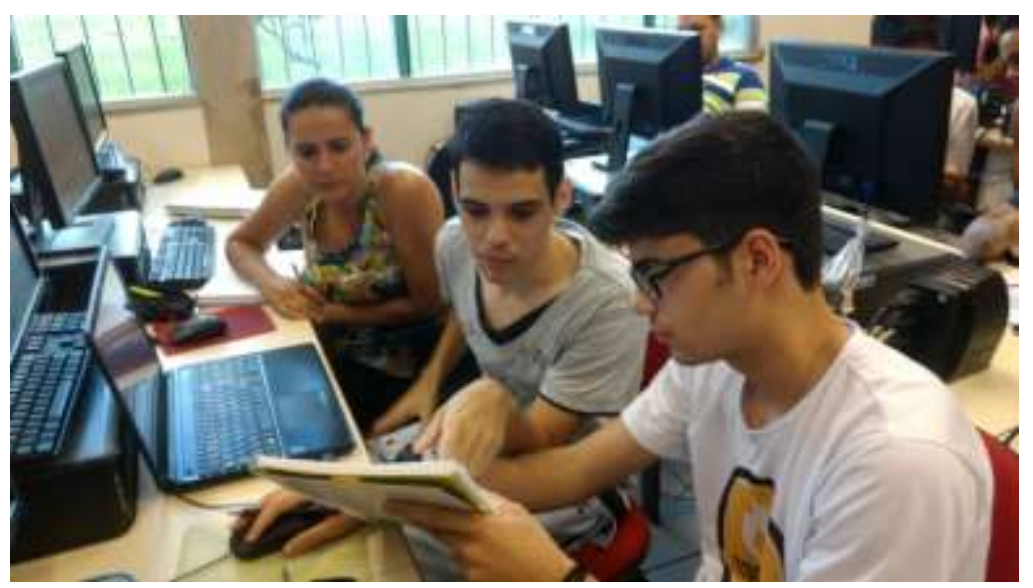

Figura 3. Participantes interagindo e elaborando a atividade prática

\subsection{Apresentação dos aplicativos de apoio}

No término da atividade prática foram apresentados os aplicativos de apoio (Plickers e Share It): suas definições, funcionalidades e benefícios pedagógicos. Além disso, foi realizada uma demonstração de uso das ferramentas com a turma.

\section{Resultados e Discussões}

Notou-se que a participação dos alunos em atividades sobre o tema não é o único fator determinante para a percepção positiva dos alunos quanto à utilização de tecnologias no 
processo de ensino- aprendizagem e pelo interesse em utilizar tais ferramentas quando forem professores, haja vista que a proporção da participação de atividades de extensão é aproximadamente $30 \%$ menor que as considerações positivas dos alunos.

Constatou-se que a mesma proporção de alunos que não tiveram a experiência de ver seus professores utilizando ferramentas tecnológicas no seu ensino básico $(66 \%)$ é a mesma de alunos que estão tendo essa experiência no ensino superior. Assim, podese discutir a possibilidade de que os professores são mais abertos a utilizar as tecnologias no ensino superior do que no ensino básico.

$\mathrm{Na}$ atividade prática da ação foi possível observar que 100\% dos grupos tiveram facilidade de produzir o plano de aula na plataforma online e elaborar suas apresentações em formato digital. Tal unanimidade aponta que os participantes possuem aptidão suficiente para desenvolver atividades utilizando ferramentas tecnológicas.

$\mathrm{Na}$ apresentação das atividades práticas, notou-se que $87 \%$ dos grupos consideraram os aplicativos apresentados como ferramentas auxiliares no processo de ensino aprendizagem, não sendo vistos, portanto, como a principal ferramenta para se ministrar um determinado conteúdo. Expressões como "A ferramenta auxilia na verificação da atividade, pois aprender escrevendo é importante", "O App serve como uma prova real do cálculo", nas apresentações deu suporte a esse resultado. Apenas um dos grupos (13\%) considerou inviável a utilização dos aplicativos em sala de aula, considerando que os alunos precisam aprender no modo tradicional.

Quanto à metodologia utilizada nos planos de aula e apresentação das propostas, $62 \%$ dos grupos optaram por inicialmente explicar as definições e elaborar exemplos e exercícios no caderno sobre o tema abordado e só assim, propor a utilização do aplicativo; $25 \%$ dos grupos propuseram uma competição entre os alunos para solucionar as fases do jogo do aplicativo e 13\%, somente explicou detalhadamente as funcionalidades do aplicativo.

Tanto na apresentação (figura 4) das propostas quanto à metodologia, os grupos, majoritariamente demonstraram comprometimento com a abordagem do conteúdo e com o método utilizado: apresentaram explicações fundamentais do conteúdo abordado de maneira didática, e só assim propuseram a atividade com o aplicativo. Considera- se que esse resultado é reflexo da afinidade dos participantes pela docência e pela matemática ( $40 \%$ e $34 \%$, respectivamente).

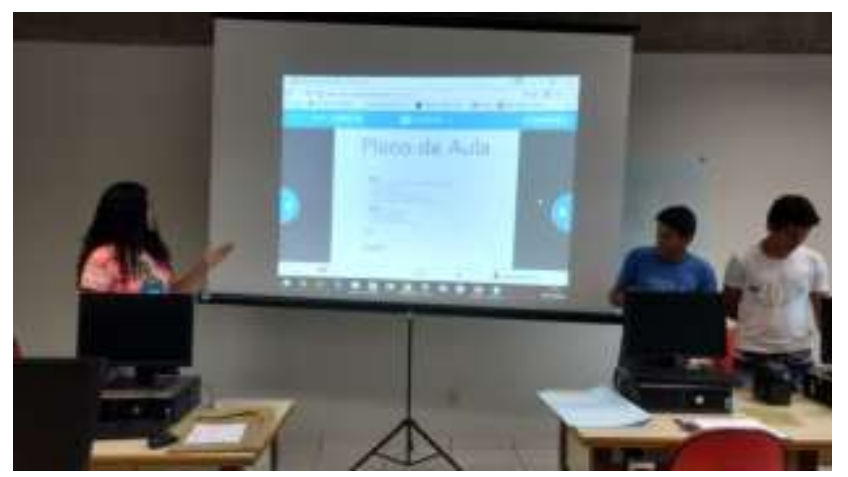

Figura 4- Alunos apres entando suas atividades práticas 
VI Congresso Brasileiro de Informática na Educação (CBIE 2017)

Anais do XXIII Workshop de Informática na Escola (WIE 2017)

Quanto à avaliação dos aplicativos pelos participantes, ainda que não tenha havido diferenças discrepantes, observa-se que o aplicativo Geogebra apresentou uma aceitação maior pelos alunos em relação à agilidade no processamento de informações (figura 5). Entretanto, não obteve a melhor nota para o fácil manuseio, ficando o $\mathrm{Mal}$ Math com a melhor nota para esse quesito. A escolha das melhores notas para esses aplicativos deve ter ocorrido em razão da grande maioria dos alunos já terem usado tais aplicativos anteriormente, conforme avaliação inicial. Além disso, o aplicativo Math Academy foi o menos aceito pelos alunos nos dois quesitos e o menos utilizado anteriormente.

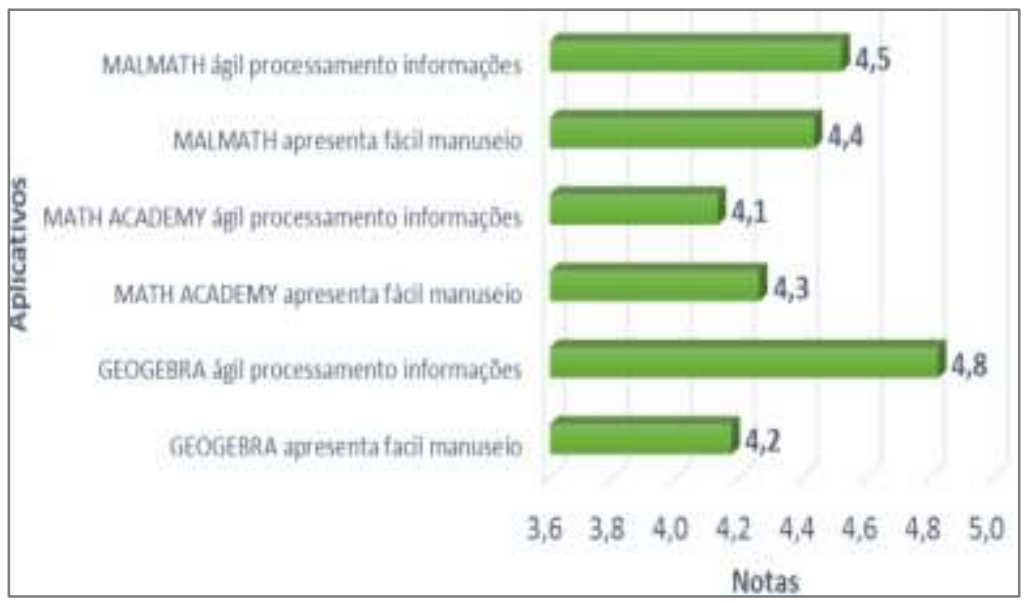

Figura 5 - Avaliação de aplicativos matemáticos

Quanto ao grau de domínio dos aplicativos após a oficina, observa-se na figura 6 que os três aplicativos não apresentaram diferenças relevantes quanto aos números de alunos satisfeitos, estes em torno de 50\%. Aproximadamente os outros 50\% mostraram ter um domínio razoável. Esses resultados evidenciam a habilidade dos participantes em utilizarem tais tecnologias como apoio pedagógico no processo de ensinoaprendizagem.

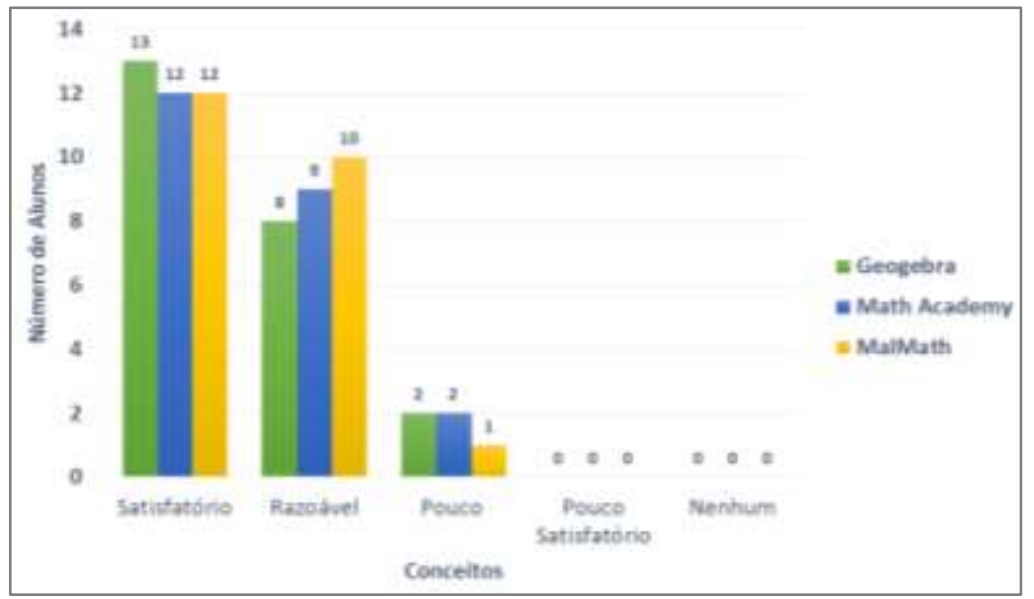

Figura 6. Grau de domínio sobre os aplicativos 
Com relação a Lei Estadual n 3.109 de 2015 (Acre) que proíbe o uso de dispositivos móveis na escola, 78\% dos participantes concordam que a lei, mesmo que permita a utilização dos dispositivos móveis para fins pedagógicos pode inibir os professores inclusive eles, a terem iniciativas de práticas com tais dispositivos em sala de aula.

Quanto à percepção da possibilidade de utilizar os aplicativos quando professores, os alunos se sentiram interessados e motivados. Unanimemente, os participantes também consideram que os aplicativos podem ser úteis na prática docente, o que demonstra a contribuição tecnológica de tais ferramentas no ensino e aprendizagem de matemática.

\section{Considerações Finais}

Conclui- se que os alunos de licenciatura em matemática estão incluídos digitalmente e aptos e engajados a utilizarem as tecnologias em dispositivos móveis como recurso didático em suas futuras práticas docente. Além disso, estabelece-se que a existência de uma Lei que proíba o uso de dispositivos móveis na sala de aula, ainda que permita a utilização para fins pedagógicos, pode ser um impedimento para que os professores tenham iniciativas em desenvolver atividades com seus alunos utilizando essas tecnologias.

Além disso, a pesquisa contribui para apresentar um método para uma atividade de formação de professores, que pode ser aprimorada e utilizada em trabalhos futuros.

Sugere-se, em trabalhos futuros, a investigação dos fatores que levam os professores a utilizarem mais os recursos tecnológicos ensino superior do que no ensino básico e um estudo para verificar quais elementos além de capacitações sobre o tema, levam os professores a se interessarem em utilizar tecnologias em suas salas de aula.

\section{Referências}

Alves, E. dos S.; Assis, C. de F. C. de; Martins, F. S. (2015). Inclusão Digital e o Ensino da Matemática em escolas públicas: Vivências no Programa Infomat/ Proext. Em: Anais do XXI Workshop de Informática na Escola (WIE 2015). DOI: 10.5753/ cbie.wie. 2015.340.

Arantes, H. Faria; Seabra, R. Duarte (2016). TME: Aplicativo M-Learning para o Estudo de Conceitos Matemáticos com Ênfase no ENEM. Em: Anais do XXVII Simposio Brasileiro de Informática na Educação (SBIE 2016). DOI: 10.5753/cbie.sbie.2016.1.

Baldissera, A. Pesquisa-Ação: Uma metodologia do "Conhecer" e do "Agir" coletivo. Sociedade em debate, Pelotas, 7 (2): 5-25, Agosto/ 2001. Disponível em:< http://revistas.ucpel.tche.br/index.php/rsd/article/viewFile/570/510>. Acesso em: 08/02/2017.

CETIC Centro de Estudos sobre as tecnologias da informação e comunicação. Tic Educação 2015. Disponível em: http://cetic.br/pesquisa/educacao/indicadores. Acesso em: 30/10/2016 
VI Congresso Brasileiro de Informática na Educação (CBIE 2017)

Anais do XXIII Workshop de Informática na Escola (WIE 2017)

Devalli, C.; Corrêa, M. M. C. (2014) Informática na Educação: uso de aplicativos para estímulo do estudo em rede. Disponível em: <http://bsi.uniriotec.br/tcc/201406De lavalliCassiano.pdf $>$. Acesso em: 30/10/2016.

DOE/AC (2015), Dispõe sobre o uso de aparelhos celulares e equipamentos eletrônicos (smartphones e tablets) nos estabelecimentos de ensino público e privado, no âmbito do Estado. Lei $n^{\circ} 3.109$ de 29/12/2015 - Arts. $\S 1^{\circ}$ e $\S 2^{\circ}$. Publicado no DOE $n^{\circ}$ 11.712 de 30/12/2015. Disponível em: http://www.al.ac.leg.br/leis/wp- content /uploads/2016/02/Lei3109.pdf. Acesso em 19/12/2016.

Ferreira, E. F. P.; Integração das tecnologias ao ensino da Matemática: percepções iniciais. Em: Anais do XIX EBRAPEM - Encontro Brasileiro de Estudantes de PósGraduação em Educação Matemática. ISSN: 2237-8448. Universidade Federal de Juiz de Fora - MG, 2015. Disponível em < http://www.ufjf.br/ebrapem 2015/anais/sessao-d-0111-tarde>. Acesso em 23/10/2016.

Marçal, E.; Andrade, R.; Rios, R. Aprendizagem utilizando dispositivos móveis com sistemas de realidade virtual. Centro Interdisciplinar de novas tecnologias na educação. v.3 n.1, maio, 2005. Disponível em:< https://inf.ufes.br/ cvnascimento/artigos/a51_realidadevirtual_revisado.pdf. Acesso em: 08/06/2017.

Nascimento, K.A.S. do; Filho, A de C.(2016). Dispositivos Móveis na educação: ensinando e aprendendo em diferentes contextos. Em: XXVII Simpósio Brasileiro de Informática na Educação (SBIE 2016). DOI: 10.5753/cbie.sbie.2016.1225.

Oliveira, L. R. de; Medina, R. D. (2007). Desenvolvimento de objetos de aprendizagem para dispositivos móveis: uma nova abordagem que contribui para a educação. Centro Interdisciplinar de Novas Tecnologias na Educação. v. 5, n 1, julho, 2007.

Oliveira, M. S. de; Fernandes, K. T. (2016) Uso de Aplicativos Móveis no Ensino da disciplina de Cálculo. Em: Congresso Regional sobre Tecnologias na Educação.

Ribeiro, D. F. (2013). Inovação e Resistência: Uma análise no ensino da matemática em uma escola pública. Dissertação- Universidade Federal de Pelotas. 98f. 18 de Outubro de 2013.

Souza, A. L.; Murta, C. A. R.; Leite, L. G. S. (2016) Tecnologia ou metodologia: aplicativos móveis na sala de aula. http://evidosol.textolivre.org/papers/2016/upload 182.pdf> Acesso em 21/10/2016.

Slomp, P.F; Machado, A.F (2013). Software Educacional Livre para Dispositivos Móveis. Disponível em: https://www.ufrgs.br/soft-livre-<edu/wiki/Software_<Edu cacional_Livre_para_Dispositivos_M\%C3\%B3veis_-_Tabela_Din\%C3\%A2mica>. Acesso em 15 de Dezembro de 2016 